\title{
Complexity-Measure-Based Sequential Hypothesis Testing for Real-Time Detection of Lethal Cardiac Arrhythmias
}

\author{
Szi-Wen Chen \\ Department of Electronic Engineering, Chang Gung University, 259 Wen-Hwa 1st Road, Kwei-Shan, Tao-Yuan 333, Taiwan
}

Received 15 June 2006; Revised 17 September 2006; Accepted 17 September 2006

Recommended by Tan Lee

A novel approach that employs a complexity-based sequential hypothesis testing (SHT) technique for real-time detection of ventricular fibrillation (VF) and ventricular tachycardia (VT) is presented. A dataset consisting of a number of VF and VT electrocardiogram (ECG) recordings drawn from the MIT-BIH database was adopted for such an analysis. It was split into two smaller datasets for algorithm training and testing, respectively. Each ECG recording was measured in a 10-second interval. For each recording, a number of overlapping windowed ECG data segments were obtained by shifting a 5-second window by a step of 1 second. During the windowing process, the complexity measure $(\mathrm{CM})$ value was calculated for each windowed segment and the task of pattern recognition was then sequentially performed by the SHT procedure. A preliminary test conducted using the database produced optimal overall predictive accuracy of $96.67 \%$. The algorithm was also implemented on a commercial embedded DSP controller, permitting a hardware realization of real-time ventricular arrhythmia detection.

Copyright (c) 2007 Szi-Wen Chen. This is an open access article distributed under the Creative Commons Attribution License, which permits unrestricted use, distribution, and reproduction in any medium, provided the original work is properly cited.

\section{INTRODUCTION}

Ventricular fibrillation (VF) and ventricular tachycardia (VT) are life-threatening cardiac arrhythmias [1]. Reduction of mortality from such cardiac causes depends on rapid detection and accurate classification of these arrhythmias. Thus, the development of accurate noninvasive techniques for identifying patients at risk of lethal arrhythmias is essential to reducing mortality from cardiac deaths. For this reason, a number of quantitative analysis techniques for electrocardiogram (ECG) arrhythmia recognition have been proposed previously [1-9]. While all these algorithms show advantages in versatile aspects of performance evaluation, some of them are still too difficult to implement and compute for defibrillators. On the other hand, for computational convenience, some algorithms utilized in either surface ECG monitoring-based automated external defibrillators (AEDs) or in implantable cardiovertor/defibrillators relied only on simple heart rate for arrhythmia detection. In fact, this might be problematic since it has been indicated that simply using heart rate as the sole feature might always unavoidably lead to a certain error rate in the detection since while both VF and VT have significantly higher rates than normal sinus rhythm, the rate range of VF overlaps with that of VT. Therefore, using the heart rate as a single feature might achieve only moderate specificity in differentiating VT from VF so that unpredictably serious consequences might result.

In this paper, a sequential hypothesis testing (SHT) algorithm in conjunction with a novel feature, dubbed complexity measure (CM), for VF and VT detection is presented. The main idea behind the approach is that the order or disorder status of a time series, such as biological signals, can be characterized using complexity. The rationale of adopting CM in this study is based on the fact that cardiac arrhythmias such as VF and VT can be thought to belong to different nonlinear physiological processes with a different complexity [7]. There actually exist a number of different approaches developed for the measure of complexity. In this study, CM was calculated using the mathematical definitions proposed by Lempel and $\mathrm{Ziv}$ [10] since it is considered as an easy-to-compute measure of complexity in a one-dimensional signal. In general, for an adequately preset window length, the method first converts a raw ECG data segment to a 0-1 string using a schematic thresholding process. Then, the $\mathrm{CM}$ value or its normalized version corresponding to that $0-1$ string can be calculated simply by successive comparisons and accumulations of the substrings. For such aspect of application, it has been indicated by previous research that the distributions of $\mathrm{CM}$ 
values, respectively, derived from the ECG signals of VF and VT groups could be perfectly separated [7]. In addition, recently a novel Lempel-Ziv complexity-based algorithm used to identify the arrhythmic pulse patterns was proposed [8].

It still remains uncertain, however, that $\mathrm{CM}$ is robust with respect to VF/VT classification. Therefore, instead of adopting a single CM value estimated from an ECG data segment with a fixed interval as the sole feature for separating VF from VT, we may speculate that the task of classification might be performed, with a degree of robustness, simply by applying the SHT technique to a feature vector composed of CM values that were successively obtained from the windowed ECG data. In fact, such a sequential technique has been applied to the development of various VF/VT detection approaches [2-4]. Our study here aimed at seeking a possible application of a robust sequential form into CM estimates to achieve higher sensitivity and specificity for VF and VT with low computational cost.

This paper is organized as follows. Descriptions of the CM calculation defined by Lempel and Ziv and the development of the proposed sequential computing method for realtime detection of VF and VT are in Section 2. Then, a performance evaluation and discussion are presented in Section 3. Section 4 gives a brief description of the hardware settings. Finally, the paper is briefly concluded by Section 5 .

\section{METHODOLOGY}

\subsection{Complexity measure}

The estimation of CM for ECG time series is reviewed in this section. Initially, an ECG data segment, denoted by $\{x(k)\}_{k=1}^{n}$, is converted into a new time sequence $\{s(k)\}_{k=1}^{n}$ composed of 0's and 1's only. The major steps of transforming the original ECG signal into the $0-1$ string are described as follows. First, the ECG signal is normalized by subtracting its mean value from every data sample $x(k)$, that is,

$$
x_{N}(k)=x(k)-\frac{1}{n} \sum_{k=1}^{n} x(k),
$$

where $x_{N}(k)$ represents the normalized ECG signal. Next, the positive and negative peak values, denoted as $P_{p}$ and $P_{n}$, respectively, are searched out. Let $N_{p}$ represent the number of data points $x(k)$ that satisfy $0<x(k)<0.1 P_{p}$ and $N_{n}$ represent the number of those that satisfy $0.1 P_{n}<x(k)<0$, a thresholding scheme can be expressed as follows [7].

(1) If $\left(N_{p}+N_{n}\right)<0.4 n \Rightarrow$ the threshold, denoted as Th, is selected as $\mathrm{Th}=0$.

(2) If $\left(N_{p}+N_{n}\right) \geq 0.4 n$ and $N_{n}<N_{p} \Rightarrow$ Th $=0.2 P_{n}$.

(3) If $\left(N_{p}+N_{n}\right) \geq 0.4 n$ and $N_{n} \geq N_{p} \Rightarrow$ Th $=0.2 P_{p}$. Finally, the normalized ECG signal $\left\{x_{N}(k)\right\}_{k=1}^{n}$ is converted into 0 -1 string $\{s(k)\}_{k=1}^{n}$ simply by

$$
s(k)= \begin{cases}1 & \text { if } x(k) \geq \mathrm{Th} \\ 0 & \text { otherwise }\end{cases}
$$

According to Lempel and Ziv's work [10], the computational algorithm of CM, denoted as $c_{n}$, is briefly described as follows. Let $S$ and $Q$ denote two strings, respectively, and let $S Q$ represent the string resulting from concatenation of $S$ and $Q$. Moreover, $S Q \pi$ represents a new string derived from $S Q$ with the last symbol deleted. Let $v(S Q \pi)$ denote the set of all different "parts" (substrings) of $S Q \pi$. Given a finite symbol sequence $\{s(k)\}_{k=1}^{n}$, the algorithm can be summarized as follows.

(1) At the beginning, $c_{n}=1, S=s(1), Q=s(2), S Q \pi=$ $s(1)$.

(2) In the midst of computation, suppose at this time that $S=s(1) s(2) \cdots s(m), Q=s(m+1) s(m+2) \cdots s(m+p)$ and $S Q \pi=s(1) s(2) \cdots s(m) s(m+1) \cdots s(m+p-1)$, two conditions are considered:

(a) if $Q \in v(S Q \pi)$ (i.e., $Q$ is a substring of $S Q \pi)$, then $c_{n}$ and $S$ remain unchanged, and meanwhile, we set

$$
Q \longleftarrow Q s(m+p+1)
$$

that is, renew $Q$ simply by padding it with the symbol $s(m+p+1)$;

(b) if $Q \notin v(S Q \pi)$ (i.e., $Q$ is not a substring of $S Q \pi)$, then we set

$$
\begin{gathered}
c_{n} \longleftarrow c_{n}+1, \quad S \longleftarrow S Q, \\
Q \longleftarrow s(m+p+1) .
\end{gathered}
$$

(3) Repeat doing step (2) until SQ $=s(1) s(2) \cdots s(n)$.

As a result, the result of $c_{n}$ gives the number of different substrings of $\{s(k)\}_{k=1}^{n}$ and it can be viewed as the measure of complexity. It can be seen from the above that there is no a priori information or parameter required to be determined and the calculation is very simple for short-term one-dimensional signals. Further, as shown by Lempel and Ziv [10], for $s(k) \in\{0,1\}$, where $k=1,2, \ldots, n$, we have

$$
\lim _{n \rightarrow \infty} c_{n}=b_{n}=\frac{n}{\log _{2}(n)}
$$

Note that in order to reduce the variation of the above measure $c_{n}$ due to its dependence on the sequence length $n$, the normalized $\mathrm{CM}$ value $C$, defined as $C=c_{n} / b_{n}$ (where $0 \leq C \leq 1$ ), was employed for VF and VT detection throughout this analysis.

A dataset consisting of 70 ECG recordings (25 VFs and 45 VTs) drawn from the MIT-BIH database was adopted for such an analysis. Each ECG recording was 10 seconds in length and the sampling frequency $f_{s}$ was $250 \mathrm{~Hz}$. The dataset was further split into two smaller datasets for algorithm training (40 ECG recordings) and testing (30 ECG recordings), respectively. In order to perform the task of VF/VT detection using the SHT, a training database consisting of 40 ECG data recordings (15 VFs and $25 \mathrm{VTs}$ ) was used for 


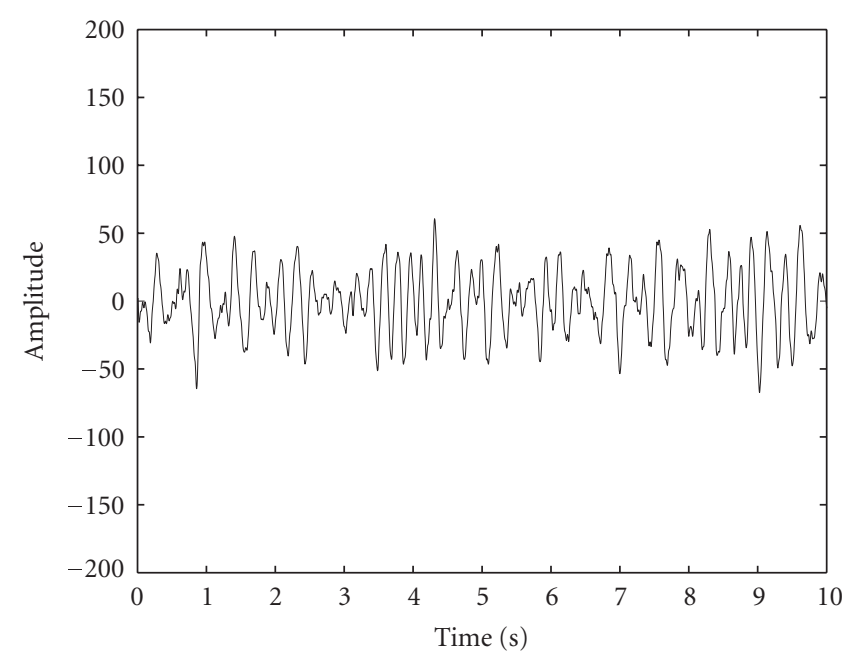

(a)

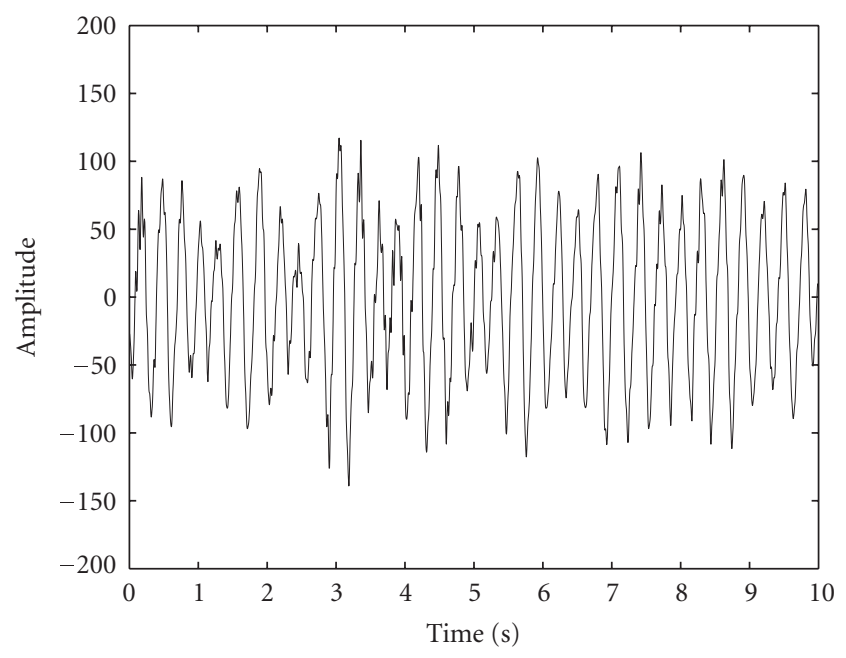

(b)

FIGURE 1: Filtered arrhythmic signals: (a) VF, (b) VT.

TABLE 1: Performance of the proposed CM-SHT method for VF and VT detection.

\begin{tabular}{l|ccccc}
\hline Mean of CM & Std. of CM & $\alpha, \beta$ values & Correct VF $(\%)$ & Correct VT $(\%)$ & Overall detection $(\%)$ \\
\hline$\mu_{\mathrm{VF}}=0.2369$ & $\sigma_{\mathrm{VF}}=0.0369$ & $0.085 \sim 0.100$ & $10 / 10=100 \%$ & $19 / 20=95 \%$ & $29 / 30 \approx 96.67 \%$ \\
$\mu_{\mathrm{VT}}=0.1641$ & $\sigma_{\mathrm{VT}}=0.0273$ & & & & \\
\hline
\end{tabular}

constructing the first- and second-order statistics. Figure 1 shows examples of the bandpass filtered VF and VT signals. The algorithm training process was performed in the following manners. A number of overlapping windowed ECG data segments were obtained by shifting a 5 -second window by a step of 1 second throughout the entire 10-second recording, thus resulting in six windowed segments for each ECG recording. For each windowed segment, a normalized CM, denoted as $C_{i}$, was calculated. The rationale for the choice of 5-second window for CM calculation is that for VF and VT the $C_{i}$ 's begin to well separate from each other when measured from 5-second ECG data segments [7]. As a result, for each ECG recording, a feature vector composed of six CM values that were, respectively, estimated from the six 5-s windowed ECG segments would result. After obtaining the CM estimates for all the ECG recordings in the training database, the means, denoted as $\mu_{\mathrm{VF}}, \mu_{\mathrm{VT}}$, and standard deviations, denoted as $\sigma_{\mathrm{VF}}, \sigma_{\mathrm{VT}}$, corresponding to both the VF and VT populations were calculated. Numerical results of these statistics are shown in Table 1. These statistics were then incorporated in the Wald-type SHT algorithm $[11,12]$.

\subsection{Sequential hypothesis testing}

The SHT algorithm implementation is described as follows. According to the numerical experimental results obtained from our analysis, we seek to discriminate the hypotheses below (see also Table 1):

$$
\begin{array}{ll}
H_{\mathrm{VF}}: \mu_{\mathrm{VF}}=0.2369, & \sigma_{\mathrm{VF}}=0.0369 ; \\
H_{\mathrm{VT}}: \mu_{\mathrm{VT}}=0.1641, & \sigma_{\mathrm{VT}}=0.0273 .
\end{array}
$$

To approach the discrimination above, a likelihood ratio test (LRT) $\Lambda$ is constructed:

$$
\Lambda\left(C_{1}, C_{2}, \ldots, C_{i}\right)=\frac{f\left(C_{1}, C_{2}, \ldots, C_{i} \mid \mathrm{VF}\right)}{f\left(C_{1}, C_{2}, \ldots, C_{i} \mid \mathrm{VT}\right)},
$$

and two thresholds, $T_{1}$ and $T_{2}$, are selected as

$$
T_{1}=\frac{1-\beta}{\alpha}, \quad T_{2}=\frac{\beta}{1-\alpha}, \quad\left(T_{1}>T_{2}>0\right),
$$

where $f$ is the conditional probability density function under the hypothesis $H_{\mathrm{VF}}$ or $H_{\mathrm{VT}}$, and $\alpha, \beta$ both represent the predetermined error probabilities; $\alpha$ is the probability of rejecting $H_{\mathrm{VT}}$ when it is true and $\beta$ is the probability of rejecting $H_{\mathrm{VF}}$ when it is true. Comparing the LRT $\Lambda$ with the two thresholds $T_{1}$ and $T_{2}$, if $\Lambda\left(C_{1}, \ldots, C_{i}\right) \geq T_{1}$ (or $\left.\Lambda\left(C_{1}, \ldots, C_{i}\right) \leq T_{2}\right)$, then the algorithm selects $H_{\mathrm{VF}}\left(\right.$ or $\left.H_{\mathrm{VT}}\right)$ and the test is terminated. If $T_{2}<\Lambda\left(C_{1}, \ldots, C_{i}\right)<T_{1}$, then the test is inconclusive. In this case, the next $\mathrm{CM}$ value $C_{i+1}$ will be incorporated into the test, that is, $\Lambda\left(C_{1}, \ldots, C_{i}, C_{i+1}\right)$ is calculated and then compared with $T_{1}$ and $T_{2}$. The above process is repeated until either a decision is finally reached, or all the CM values have been incorporated in the LRT but still no decision is made. 


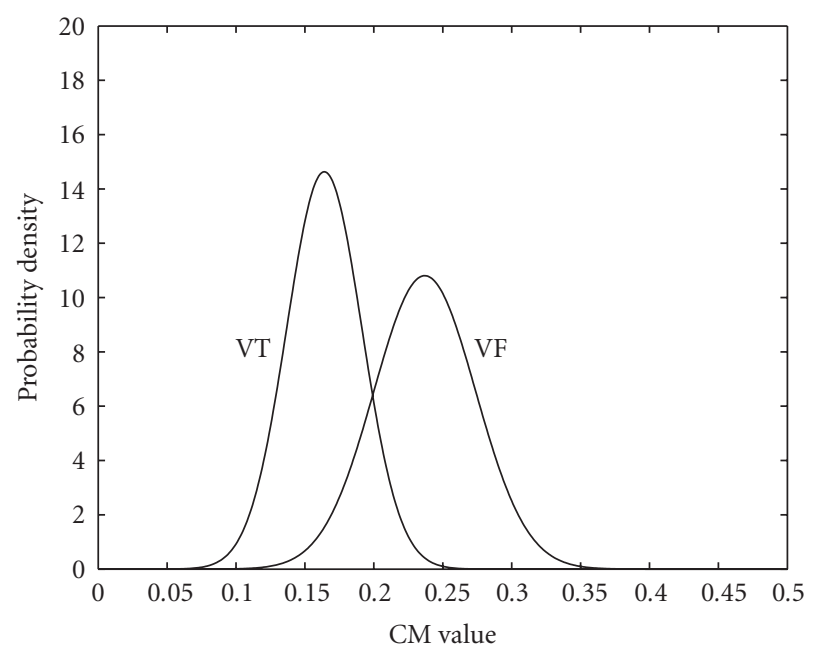

FIGURE 2: The modeled Gaussian probability density functions of $\mathrm{CM}$ for VF and VT, respectively.

According to the numerical results obtained from the training process, the $\mathrm{CM}$ values obtained from the VF and VT populations can be well modeled by Gaussian distributions with different means and standard deviations, as shown in Figure 2. Therefore, the logarithmic form of (7) for a test comprising $m$ successive CM values estimated from an ECG data recording is expressed as

$$
\begin{aligned}
\ln \left(\Lambda_{m}\right)= & \ln \left[\frac{f\left(C_{1}, C_{2}, \ldots, C_{m} \mid \mathrm{VF}\right)}{f\left(C_{1}, C_{2}, \ldots, C_{m} \mid \mathrm{VT}\right)}\right] \\
= & \ln \left[\frac{\prod_{i=1}^{m}\left(1 / \sqrt{2 \pi \sigma_{\mathrm{VF}}^{2}}\right) \exp \left(-\left(C_{i}-\mu_{\mathrm{VF}}\right)^{2} / 2 \sigma_{\mathrm{VF}}^{2}\right)}{\prod_{i=1}^{m}\left(1 / \sqrt{2 \pi \sigma_{\mathrm{VT}}^{2}}\right) \exp \left(-\left(C_{i}-\mu_{\mathrm{VT}}\right)^{2} / 2 \sigma_{\mathrm{VT}}^{2}\right)}\right] \\
= & m \ln \left(\frac{\sigma_{\mathrm{VT}}}{\sigma_{\mathrm{VF}}}\right)+\frac{1}{2 \sigma_{\mathrm{VT}}^{2}} \sum_{i=1}^{m}\left(C_{i}-\mu_{\mathrm{VT}}\right)^{2} \\
& -\frac{1}{2 \sigma_{\mathrm{VF}}^{2}} \sum_{i=1}^{m}\left(C_{i}-\mu_{\mathrm{VF}}\right)^{2} .
\end{aligned}
$$

Comparing $\ln \left(\Lambda_{m}\right)$ with $\ln \left(T_{1}\right)$ and $\ln \left(T_{2}\right)$, a decision rule at the $m$ th stage can be then formulated as

$$
\begin{aligned}
& g\left(C_{1}, \ldots, C_{m}\right) \geq s_{1}, \Longrightarrow H_{\mathrm{VF}} \\
& \leq s_{2}, \Longrightarrow H_{\mathrm{VT}} ; \\
& \text { otherwise } \Longrightarrow \text { continue testing, }
\end{aligned}
$$

where

$$
\begin{aligned}
g\left(C_{1}, \ldots, C_{m}\right) & =\frac{1}{\sigma_{\mathrm{VT}}^{2}} \sum_{i=1}^{m}\left(C_{i}-\mu_{\mathrm{VT}}\right)^{2}-\frac{1}{\sigma_{\mathrm{VF}}^{2}} \sum_{i=1}^{m}\left(C_{i}-\mu_{\mathrm{VF}}\right)^{2}, \\
s_{1} & =2 m \ln \left(\frac{\sigma_{\mathrm{VF}}}{\sigma_{\mathrm{VT}}}\right)+2 \ln \left(\frac{1-\beta}{\alpha}\right), \\
s_{2} & =2 m \ln \left(\frac{\sigma_{\mathrm{VF}}}{\sigma_{\mathrm{VT}}}\right)+2 \ln \left(\frac{\beta}{1-\alpha}\right) .
\end{aligned}
$$

The test above is repeated until a decision (VF or VT) is finally made. A block diagram of the CM-SHT algorithm for VF and VT detection described above is also depicted in Figure 3.

\section{PERFORMANCE EVALUATION AND DISCUSSION}

In this section, a software-based performance evaluation of the proposed algorithm is presented. A subset of the MIT$\mathrm{BIH}$ database consisting of 30 recordings (10 VFs and 20 VTs) was adopted as a testing database for a preliminary test in this study. Each recording was 10 seconds in length and sampled by the rate of $250 \mathrm{~Hz}$. Initially, the ECG signals were preprocessed by digital bandpass filtering $(2-30 \mathrm{~Hz})$ for removal of baseline drift, motion artifacts, and $60 \mathrm{~Hz}$ powerline interference. The digital bandpass filter is formed by a cascade of the fourth-order Butterworth lowpass and highpass filters.

The numerical results are listed in Table 1. It is observed from the table that while only one VT episode was misclassified into the VF class, none of VF episodes were improperly identified as VT. Consequently, the proposed CM-SHT approach optimally produced near $97 \%$ overall predictive accuracy for both arrhythmic events, which is quite promising. Such a predictive accuracy is better than that achieved by a previous SHT-based algorithm proposed by Chen et al. $[3,4]$. They employed a feature called "blanking variability" (BV) as the basis for VF/VT discrimination and showed that their method only achieved 95\% overall detection rate.

Demonstrative examples for quantitatively showing the calculations of the CM and the SHT for one episode of VF and VT (as shown in Figures 1(a) and 1(b)) are provided in Tables 2 and 3, respectively. Moreover, illustrations in Figure 4 also show the sequential detection tracings for demonstrating the decision-making processes corresponding to both examples. It should be noted that according to the detection results achieved in this study, almost all the ventricular arrhythmias can be properly identified within (mostly much faster than) 10 seconds. This is desirable and suitable for clinical purpose. All in all, the preliminary results actually indicated that the proposed approach might possess a good and hopeful potential for discriminating VF from VT.

In addition, it has been known that there exists a tradeoff between the error probabilities and the time steps or number of tests in the Wald-type hypothesis testing algorithm. In general, the larger the error probability is set, the faster the decision is made, and vice versa. Both (12) and (13) explain such a tradeoff effect. From (12) and (13), we see that the upper decision bound $s_{1}$ and lower decision bound $s_{2}$ will be getting closer as both $\alpha$ and $\beta$ increase; on the contrary, $s_{1}$ and $s_{2}$ will be further apart when $\alpha$ and $\beta$ decrease. That is, setting a larger error probability would result in a narrower nodecision region so that the tracings of function $g\left(C_{1}, \ldots, C_{m}\right)$ would be easier to leave this region for reaching a decision of VF or VT, implying that a decision might be reached more quickly. Figure 5 gives an interpretative illustration for this fact. 


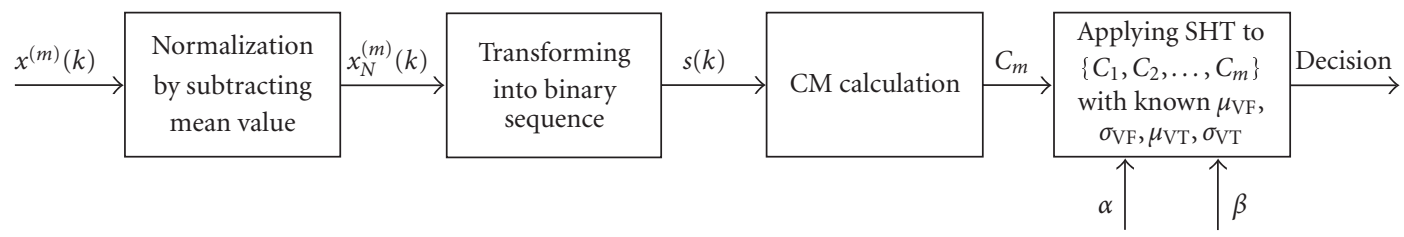

FIGURE 3: Block diagram of the detection algorithm. $x^{(m)}(k)$ denotes the $m$ th windowed ECG segment.

TABLE 2: A demonstrative example for quantitatively showing the calculations of the CM and the SHT for the VF episode shown in Figure 1(a). One may see that the episode is properly specified as VF at the fourth testing stage (i.e., number of SHT tests $=4$ ).

\begin{tabular}{|c|c|c|c|c|c|}
\hline Stage no. $(m)$ & $\mathrm{CM}\left(C_{m}\right)$ & $s_{1}$ & $g\left(C_{1}, \ldots, C_{m}\right)$ & $s_{2}$ & Test \\
\hline$m=1$ & $C_{1}=0.1975$ & 5.3588 & $g\left(C_{1}\right)=0.3672$ & -4.1463 & $s_{2}<g<s_{1}$ \\
\hline$m=2$ & $C_{2}=0.2222$ & 5.9650 & $g\left(C_{1}, C_{2}\right)=4.7581$ & -3.5401 & $s_{2}<g<s_{1}$ \\
\hline$m=3$ & $C_{3}=0.2058$ & 6.5712 & $g\left(C_{1}, C_{2}, C_{3}\right)=6.3837$ & -2.9339 & $s_{2}<g<s_{1}$ \\
\hline$m=4$ & $C_{4}=0.2304$ & 7.1775 & $g\left(C_{1}, C_{2}, C_{3}, C_{4}\right)=12.2816$ & -2.3276 & $g \geq s_{1}(\mathrm{VF})$ \\
\hline$m=5$ & - & 7.7837 & - & -1.7214 & - \\
\hline$m=6$ & - & 8.3899 & - & -1.1152 & - \\
\hline
\end{tabular}

TABLE 3: A demonstrative example for quantitatively showing the calculations of the CM and the SHT for the VT episode shown in Figure 1(b). One may see that the episode is properly specified as VT at the fifth testing stage (i.e., number of SHT tests $=5$ ).

\begin{tabular}{|c|c|c|c|c|c|}
\hline Stage no. $(m)$ & $\mathrm{CM}\left(C_{m}\right)$ & $s_{1}$ & $g\left(C_{1}, \ldots, C_{m}\right)$ & $s_{2}$ & Test \\
\hline$m=1$ & $C_{1}=0.1893$ & 5.3588 & $g\left(C_{1}\right)=-0.8084$ & -4.1463 & $s_{2}<g<s_{1}$ \\
\hline$m=2$ & $C_{2}=0.2058$ & 5.9650 & $g\left(C_{1}, C_{2}\right)=0.8172$ & -3.5401 & $s_{2}<g<s_{1}$ \\
\hline$m=3$ & $C_{3}=0.1811$ & 6.5712 & $g\left(C_{1}, C_{2}, C_{3}\right)=-1.0840$ & -2.9339 & $s_{2}<g<s_{1}$ \\
\hline$m=4$ & $C_{4}=0.1893$ & 7.1775 & $g\left(C_{1}, C_{2}, C_{3}, C_{4}\right)=-1.8924$ & -2.3276 & $s_{2}<g<s_{1}$ \\
\hline$m=5$ & $C_{5}=0.1564$ & 7.7837 & $g\left(C_{1}, C_{2}, C_{3}, C_{4}, C_{5}\right)=-6.5747$ & -1.7214 & $g \leq s_{2}(\mathrm{VT})$ \\
\hline$m=6$ & - & 8.3899 & - & -1.1152 & - \\
\hline
\end{tabular}

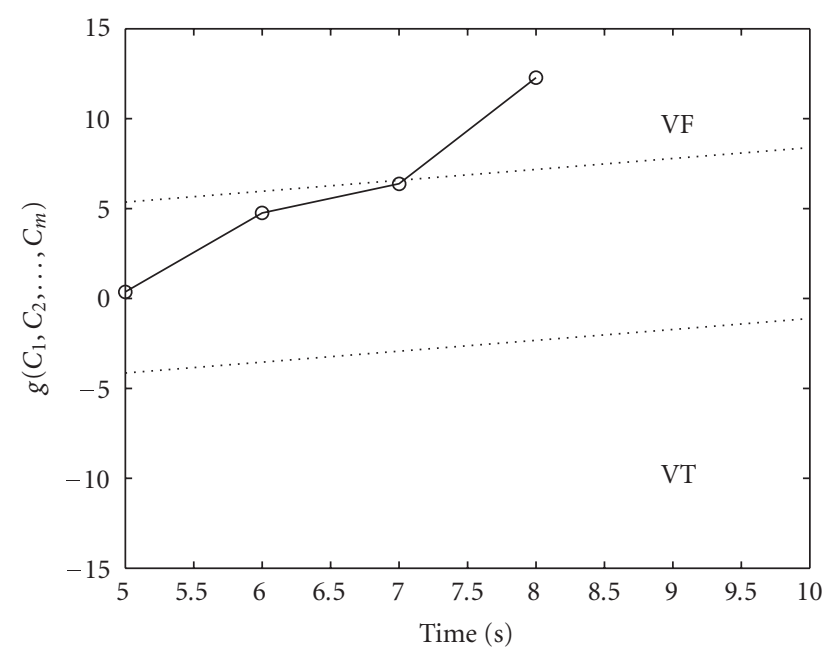

(a)

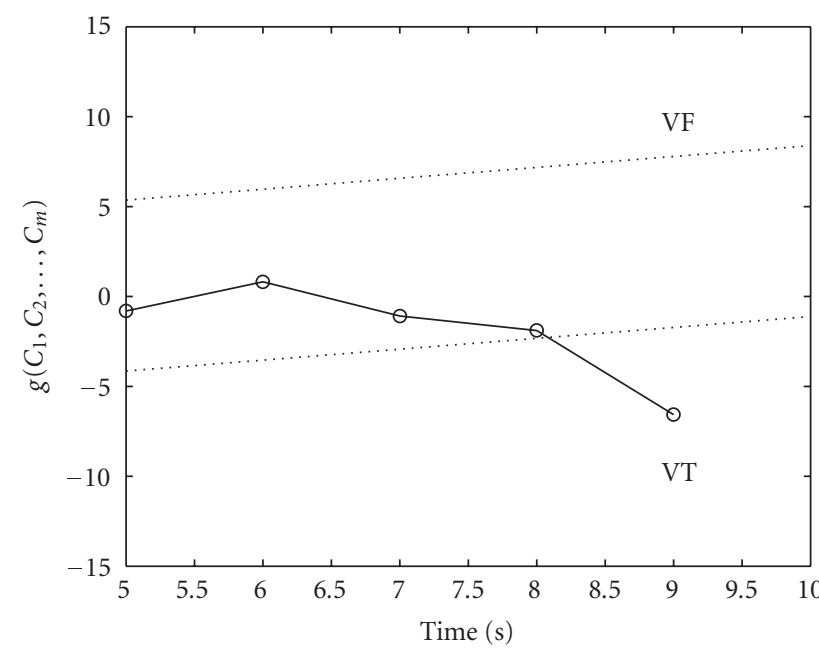

(b)

FIgUre 4: Sequential detection tracings for (a) VF, (b) VT, $\alpha=\beta=0.085$. Note that here (a) and (b) are plotted according to the numerical results as provided by Tables 2 and 3, respectively. 


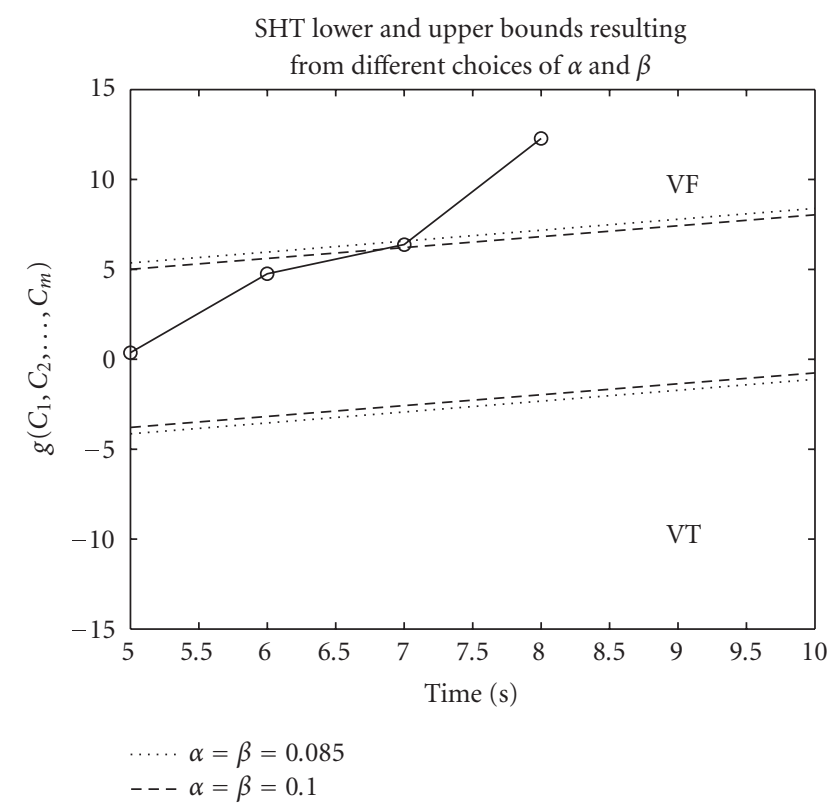

Figure 5: Tracings of the function $g\left(C_{1}, \ldots, C_{m}\right)$. It can be seen from the figure that by setting $\alpha=\beta=0.085$ (depicted by dotted lines), a decision was reached right after four $\mathrm{CM}$ values were included for testing (number of tests $=4$, time taken for decision $=8$ seconds); if choosing $\alpha=\beta=0.100$ (depicted by the dashed lines), a decision (but the same decision as the case above not guaranteed) would be achieved more quickly (number of tests $=3$, time taken for decision $=7$ seconds). This is because the latter would result in a narrower no-decision region, thus the tracings of $g\left(C_{1}, \ldots, C_{m}\right)$ might leave this region with a higher probability.

On the other hand, it should be also noted that the results obtained were not critically dependent on the choices for $\alpha$ and $\beta$, indicating a degree of robustness in the performance of the algorithm. Moreover, in order to understand how the SNR affects the performance of the proposed classifier, a numerical experiment was further performed in this study. Here, we had taken a case where the simulated ECG measurement was a composite signal of a noise-free ECG and a zero-mean white Gaussian noise with variance $\sigma^{2}$. Simulated noise-free ECG signals were generated by applying bandpass filtering (with the passband of $2-30 \mathrm{~Hz}$ ) to all the ECG episodes in the testing database. Also, different noise levels, that is, the values of $\sigma$, were respectively determined by a set of given SNR values from 0-30 dB. Consequently, each of the simulated ECG signals was finally composed by adding a noise signal to a noisefree ECG. Then, performances were evaluated on all these simulated ECG measurements. The overall detection rates at different SNR values are listed in Table 4 and are also presented in Figure 6. Observing the results in Table 4 and Figure 6, one may see that the optimal overall detection rate achieved by the proposed classifier could be achieved at SNR $28 \mathrm{~dB}$. Meanwhile, it is also revealed from Table 4 that the level of acceptable SNR should be at least $14 \mathrm{~dB}$ or more, since promising performance in VF/VT classification
TABLE 4: SNR versus the overall detection accuracy.

\begin{tabular}{c|c}
\hline SNR $(\mathrm{dB})$ & Overall detection $(\%)$ \\
\hline 0 & 33.33 \\
2 & 33.33 \\
4 & 46.67 \\
6 & 63.33 \\
8 & 70 \\
10 & 70 \\
12 & 83.33 \\
14 & 93.33 \\
16 & 93.33 \\
18 & 93.33 \\
20 & 93.33 \\
22 & 93.33 \\
24 & 93.33 \\
26 & 93.33 \\
28 & 96.67 \\
30 & 96.67 \\
\hline
\end{tabular}

accuracy $(93.33 \%)$ could be still achieved by the proposed algorithm.

Further, note that as mentioned previously, all the numerical experimental results were obtained from the $250 \mathrm{~Hz}$ sampled ECG data. In general, for arrhythmia detection, selecting the ECG sampling rate within $[200,250] \mathrm{Hz}$ is appropriate $[2-5,7]$. It is, however, interesting to speculate that if one can get the similar results with a smaller sampling frequency, the embedded real-time detection of lethal cardiac arrhythmias may be implemented in a lower powerconsuming microprocessor. Table 5 shows the performance of the proposed CM-SHT method achieved when the sampling rate is reduced to $125 \mathrm{~Hz}$. It is revealed from Table 5 that similar to the previous case, there was only a misclassified VT episode (i.e., the VT episode was misclassified into the VF class) and none of VF episodes were improperly identified as VT; however, in this case there was an inconclusive episode seen in the algorithm evaluation, thus resulting in about $93.33 \%$ overall predictive accuracy. Nevertheless, such a performance can be still considered promising and acceptable.

Although one may speculate that employing a higher sampling rate might tax the capability of microprocessors for online real-time processing, yet it is expectable that with the steady improvement in microprocessor speed and computing power, this will not cause too much trouble. Therefore, in the practical hardware implementation of the proposed algorithm here, the sampling rate was still selected as $250 \mathrm{~Hz}$. Descriptions of hardware settings will be in the next section.

\section{HARDWARE SETTINGS}

The algorithm was also implemented on a commercial embedded DSP controller built by Spinel Technology Corporation, Taiwan (STC32 DSP Board). The onboard CPU is 
TABle 5: Performance of the proposed CM-SHT method when the sampling rate is reduced to $125 \mathrm{~Hz}$.

\begin{tabular}{l|ccccc}
\hline Mean of CM & Std. of CM & $\alpha, \beta$ values & Correct VF $(\%)$ & Correct VT $(\%)$ & Overall detection $(\%)$ \\
\hline$\mu_{\mathrm{VF}}=0.3912$ & $\sigma_{\mathrm{VF}}=0.0577$ & $0.020 \sim 0.025$ & $10 / 10=100 \%$ & $18 / 19 \approx 94.74 \%$ & $28 / 30 \approx 93.33 \%$ \\
$\mu_{\mathrm{VT}}=0.2544$ & $\sigma_{\mathrm{VT}}=0.0463$ & & & (one is inconclusive) & \\
\hline
\end{tabular}

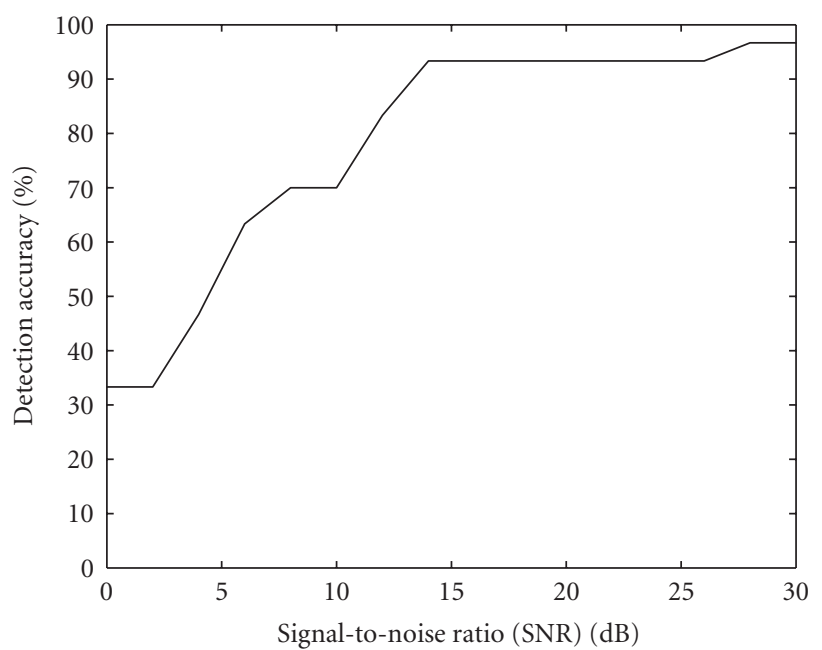

FiguRE 6: SNR versus the overall detection accuracy.

a 32-bit, $50 \mathrm{MHz}$ floating-point digital signal processor (TI TMS320C32). An environment of the STC32 software development can be formed using the C3x code composer and TI XDS510 emulator. In this hardware setting, raw ECG signals were $\mathrm{A} / \mathrm{D}$ converted with a resolution of $12 \mathrm{bits}$ every 4 milliseconds (sampling frequency $f_{s}=250 \mathrm{~Hz}$ ). The TMS320C32 was then performing the bandpass prefiltering $(2-30 \mathrm{~Hz})$, the complexity calculations, as well as the subsequent SHT for the task of decision making. Further, to continuously monitor the detection results, a display interface was also accomplished by Borland $\mathrm{C}++$ Builder. It displayed the original ECG signals, CM values, and the final detection results in PC. Detection results were indicated by three flash colored lights as follows-green: "normal sinus rhythm," yellow: "VT," and red: "VF." Note that the processing time of lethal arrhythmia detection task was adequately short for beat to beat, permitting a real-time processing.

\section{CONCLUSION}

In this paper, a real-time ventricular arrhythmia detection algorithm is proposed. In this algorithm, a sequential hypothesis testing in conjunction with a complexity-measure-based feature for VF and VT detection plays the central role of the novel algorithm. As for its hardware realization, the algorithm was also successfully implemented on a commercial DSP board, functioning as a real-time ventricular arrhythmia diagnostic system. To sum up, in addition to being principally different from the time- and frequency-domain approaches, the novel CM-SHT method had many advantages over the conventional detection algorithms in many aspects such as its reliability, simplicity, and feasibility in clinical use as well as in practical AED implementation.

\section{ACKNOWLEDGMENT}

This work was supported by the National Science Council, Taiwan, under Contract NSC 92-2220-E-182-003.

\section{REFERENCES}

[1] M. E. Cain, H. D. Ambos, J. Markham, B. D. Lindsay, and R. M. Arthur, "Diagnostic implications of spectral and temporal analysis of the entire cardiac cycle in patients with ventricular tachycardia," Circulation, vol. 83, no. 5, pp. 1637-1648, 1991.

[2] N. V. Thakor, Y.-S. Zhu, and K.-Y. Pan, "Ventricular tachycardia and fibrillation detection by a sequential hypothesis testing algorithm," IEEE Transactions on Biomedical Engineering, vol. 37, no. 9, pp. 837-843, 1990.

[3] S.-W. Chen, P. M. Clarkson, and Q. Fan, "A sequential technique for cardiac arrhythmia discrimination," Journal of Electrocardiology, vol. 28, supplement 1, p. 162, 1995.

[4] S.-W. Chen, P. M. Clarkson, and Q. Fan, "A robust sequential detection algorithm for cardiac arrhythmia classification," IEEE Transactions on Biomedical Engineering, vol. 43, no. 11, pp. 1120-1125, 1996.

[5] S.-W. Chen, "A two-stage discrimination of cardiac arrhythmias using a total least squares-based Prony modeling algorithm," IEEE Transactions on Biomedical Engineering, vol. 47, no. 10, pp. 1317-1327, 2000.

[6] K. L. Ripley, T. E. Bump, and R. C. Arzbaecher, "Evaluation of techniques for recognition of ventricular arrhythmias by implanted devices," IEEE Transactions on Biomedical Engineering, vol. 36, no. 6, pp. 618-624, 1989.

[7] X.-S. Zhang, Y.-S. Zhu, N. V. Thakor, and Z.-Z. Wang, "Detecting ventricular tachycardia and fibrillation by complexity measure," IEEE Transactions on Biomedical Engineering, vol. 46, no. 5, pp. 548-555, 1999.

[8] L. Xu, D. Zhang, K. Wang, and L. Wang, "Arrhythmic pulses detection using Lempel-Ziv complexity analysis," EURASIP Journal on Applied Signal Processing, vol. 2006, Article ID 18268, 12 pages, 2006.

[9] D. A. Coast, R. M. Stern, G. G. Cano, and S. A. Briller, "An approach to cardiac arrhythmia analysis using hidden Markov models," IEEE Transactions on Biomedical Engineering, vol. 37, no. 9, pp. 826-836, 1990.

[10] A. Lempel and J. Ziv, "On the complexity of finite sequences," IEEE Transactions on Information Theory, vol. 22, no. 1, pp. 75-81, 1976. 
[11] K. Fukunaga, Introduction to Statistical Pattern Recognition, Academic Press, New York, NY, USA, 1990.

[12] A. J. Wald, Sequential Analyses, Dove, New York, NY, USA, 1947.

Szi-Wen Chen was born in Taipei, Taiwan, 1966. He received the B.S. degree from National Taiwan University, and the M.S. degree from the Ohio State University (OSU), in 1989 and 1993, respectively, both are in electrical engineering. Since April 1994, he had been with the Biomedical Engineering Center at OSU as a Graduate Research Associate and received his Ph.D. degree in biomedical engineering in August 1997. After then, he joined the Gait Analysis Labora-

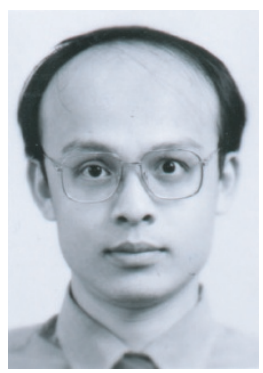
tory at OSU as a Postdoctoral Researcher. Since August 2000, he has been with the Department of Electronic Engineering as an Assistant Professor at Chang Gung University (CGU) in Taiwan, where he is also the Head of the VLSI-DSP and Bioelectronic Laboratory. His research interests are mostly in signal processing and related areas, including biomedical signal processing, artificial neural network, and pattern recognition techniques with applications to the development of medical diagnostic algorithm, embedded systems, and VLSI-DSP architectural designs. 